\title{
Programming for Vehicle Disc Pad Static $\mu$ Test by Dynamometer
}

\author{
Meizhi Huang \\ Wuhan Huaxia Uninveristy of Technology, 430056
}

\begin{abstract}
Disc pad for vehicle rear axle application has one critical requirement -- static $\mu$. Static $\mu$ is the key factor for vehicle parking on slop with hand braking only. How to simulate vehicle hand braking and rear axle working principle, programming for vehicle disc pad static $\mu$ test by Dynamometer is necessary for rear disc pad development and validation.

Key words: programming, disc pad, rear axle, static $\mu$, Dynamometer
\end{abstract}

\section{Introduction}

Vehicle disc pads are import parts in brake system of Automotive. It not only influences the vehicle braking performance, but also links to the safety for driver and passengers [1].

Disc pads are installed in caliper as below figure 1, and the two pieces brake pads will hold the rotor under hydraulic pressure from caliper when driver steps on the brake pedal [2]. 


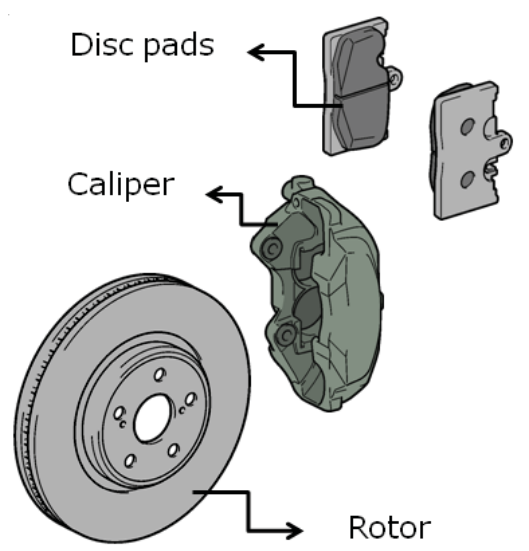

Figure 1. disc pads and brake components

For rear axle application, the disc pads will take the responsibility of stopping the running car together with front axle disc pads. Besides it, rear disc pads also take the responsibility of parking car on slop if we apply hand brake only. In this case, static $\mu$ is important performance for rear disc pads [3].

Normally, we can install the rear disc pads in vehicle and test the parking performance with hand brake only at different temperature, different slop. However, we can not have the quantitative evaluation for static $\mu$, vehicle test will take long time and high cost as well.

Programming according to the requirement and test static $\mu$ by Dynamometer will standardize the test. It will benefit rear disc pad development and performance evaluation.

\section{Experiment}

This test is to evaluate the effectiveness of the brake performance of a brake assembly when subjected to repeated parking tests with increasing and decreasing initial temperatures in a manner equivalent to the vehicle test.

The test is conducted on a dynamometer, which has the facility to both drive the brake rotor to warm up and run an incrasing torque ramp untial the shaft turns slowly to measure the static torque $[4,5]$.

By simulating the max. clamping force via applying a hydraulic pressure. In order to calculate the correct static friction value, the brake adjuster has to be dismantled in a manner that the brake adjustment will lose its function. The brake had to be designated for a

By generating the max clamping force via cable. The brake assembly should be tested at its "normal" running clearance, the clearance that occurs when adjustment due to brake operation had been completed. The rotor assembly should be tested as received from the manufacture process. 


\section{Programming for simulation by Dynamometer $[6,7,8]$.}

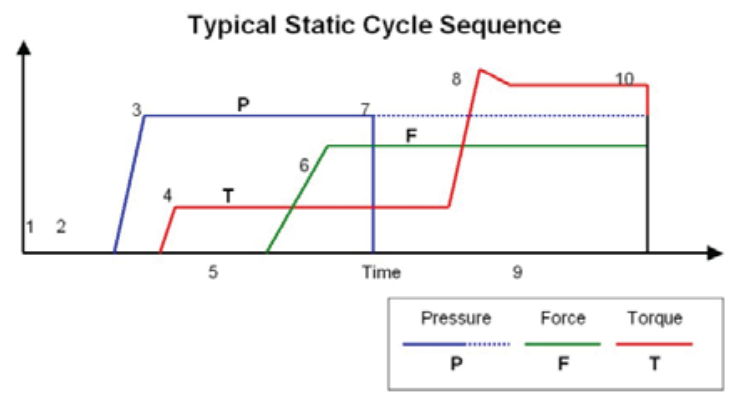

3.1.1 Sequence Overview

"Static Cycles" are cycles in which the static (very low speed) output of a braking system is measured. Static properties of brakes are usually measured to simulate how the brake will perform with the parking brake engaged, or simply the static friction level of the service brake.

Service brake and parking brake operation can be combined in this cycle to provide a closer simulation to actual vehicle performance.

"Cycles" started by using the "Run Static" (run_static) statement in a main script. The cycle algorithm (logic) is run according to the setpoints that were set in the script, prior to referencing the "Run Static" statement.

The sequence outlined below references quoted expressions, for example "Parking Brake Force Level Setpoint" that describe the parameter or event in discussion. Whenever a new setpoint is introduced (typically by a quotation), it will be followed by a the actual script function used to program the dynamometer, for example $\mathrm{pb}$ force level(Val:lb:=100).

Cycle checks to see what type of static torque system the machine is configured to use. At this point there are two basic types of static torque systems

Stroker Type - Uses air or hydraulic cylinders and a lever arm clutched to the shaft. The control system inputs pressure to the cylinders to generate torque at the brake. This type of control system works fine for most situations, but is limited by the total degrees of rotation.

Continuous Rotation Type - Uses a motor, worm-gear, clutch mechanism to generate torque at the brake. This system can rotate continuously.

The static torque system is engaged. Once proper engagement is complete, the cycle continues. If the system cannot be engaged, the cycle will shut off and an error message will be displayed.

If the static torque system is a "stroker" type, the cylinders and lever are moved (with the brake off) in accordance with the "Drive Direction Setpoint" (drive_orientation(val:= drive_orientation_forward)) so that the system is ready for forward or reverse torque.

3.1.3 Wait for Cycle Criteria (Sequence Point 2)

The dynamometer will wait in this state until the following requirements are satisfied. 
The "Controlling Temperature Channel Value" is at or below the "Initial Temperature Setpoint" (initial_temp(val:F:= 80)) or

The "Delta Temperature Setpoint" (delta_temp(val:^F:=10)) is greater than zero and the "Controlling Temperature Channel Value" is at or below the "Initial Temperature Setpoint" PLUS the "Delta Temperature Setpoint".

If the "Pressure Level Setpoint" (pressure level(Val:psi:= 500)) is greater than zero then the service brake is applied at the specified pressure level.

"Low Torque" (stq_low_torque_sp(val:\%:=25)) is applied to the brake from the static torque system.

If the "Static Service Brake Mode" (stq_svcbrk_mode(val:= stq_svcbrk_mode_svc_THEN_park)) is "Service Brake THEN Park" the system will wait with service brake applied at "Low Torque" for "Static Torque Hold Time" (stq_hold_time(val:sec:=5)).

If the "Parking Brake Force Setpoint" (pb_force_level(val:lb:= 100)) is greater than zero, then the parking brake is applied at this level. level.

The cycle waits until the "Parking Brake Force" is stable and at the specified

If the "Static Service Brake Mode" is "Service Brake THEN Park" the service brake is released or If the "Static Service Brake Mode" is "Service Brake WITH Park" the service brake and parking brake remain on at "Low Torque" until "Static Torque Hold Time" is exceeded.

"High Torque" (stq_hi_torque_sp(val:\%:=100)) is applied to the brake from the static torque system.

3.1.10 Wait for Release Criteria (Sequence Point 9)

The cycle will remain in its current state until any of the following conditions occur:

The time at "High Torque" exceeds the "Static Torque Apply Time" (stq_apply_time $($ val:sec:=30)).

If the system is a stroker type and the limits are reached on the cylinders.

If the system has angular position and the position exceeds the "Static Torque Angle Setpoint" (stq_angle_sp(val:degrees:=90)).

The parking brake is turned off if applied.

The service brake is turned off if applied.

The static torque system releases torque.

After the data is stored for the brake apply, the "Number of Cycles Setpoint" (number_of_cycles(val:=10)) is decremented.

When the number of cycles reaches zero, the static torque system is disengaged. Once proper disengagement is complete, the cycle exits. If the system cannot be disengaged, the cycle will shut off and an error message will be displayed.

The static cycle will run in a few different modes based on the setpoints used:

If the parking brake force setpoint is zero, the cycle will run service brake static only.

If the service brake pressure setpoint is zero, the cycle will run parking brake static only. 
If the service brake mode is "service then park brake" then the service brake gets released shortly after the park brake is applied.

If the service brake mode is "service with park brake" then the service brake remains on when the park brake is applied.

Quite often, the "service then park brake" mode is used for static cycles because the sequence follows what we normally do in a vehicle. That is, stop the car with the service brake, and while hydraulic pressure is applied, engage the parking brake, then release the service brake.

Some dynamometers utilize a locking mechanism on the parking brake cable puller. The objective is to let the puller get the cable load up to the setpoint, and then to lock the cable in place. Systems which incorporate this feature use the "Parking Brake Auto Lock Setpoint" (pb_auto_lock(val:= on)).

This system was designed to more closely simulate the actual events in the vehicle. Most all vehicles have a locking mechanism that holds the load on the parking brake cable. However, as the car moves, the cable lock doesn't compensate for any slack created in the cable. The locking type parking brake cable pullers simulate this action. Systems without cable locking "float" and maintain the load on the cable no matter what the brake does.

\section{Conclusion}

Through above programming and combine with dedicated test procedure, can simulate the static $\mu$ test for rear disc pad of vehicle in different temperature, different pressure condition.

\section{Reference}

[1] M.G. Jaacko, P.H.S Tang and S.K.Rhee,

Automotive friction materials evolution during the past decade, wear, 100(1984) $503 \sim 515$.

[2] Hartmut Hetzler and Wolfgang Seemann, Friction modes in low frequency disc-brake noise-experimental results and implications on modeling

[3] Katsuya Okayama, Hirokuk Fujikawa, Tatsuhisa Kubota and Kenju Kakihara, A study on rear disc brake groan noise immediately after stopping, 2005, SAE, technical paper

[4] P.H.S.Tsang, M.G.Jacko and S.K. Rhee, comparison of chase and inertial brake dynamometer testing for automotive materials, wear, 103(19850 217 232

[5]H. Jang, J.S.Lee, J.W. Fash, Compositional effects of the brake friction material on creep groam phenomena, wear 251(2001) 1477 1483.

[6]Static torque effects of temperature and load on HR caliper, S-17610200, technical specification, TRW Automotive braking system.

[7] Static $\mu$ tet procedure for brake pads, 0204Y01353-AA, Chassis Brake Interntational

[8] Prolink control system, Link Engineering company 
$\begin{array}{llr}\text { Name } & : & \text { Huang mei zhi, } \\ \text { Date of Birth } & : & \text { 1979-12, } \\ \text { Gender } & : & \text { Female }\end{array}$

Nationality: Han

Native place: Hubei Jingshan

Organization: Wuhan Huaxia University Of Technology

Degree: Master

Professional Title: Lecturer

Research direction:Computer control technology

Address

No.589 GuanShan Road, East lake high technology development zone, Wuhan, H ubei, China, 430070 